\title{
Control of Electron-State Coupling in Asymmetric Ge/Si-Ge Quantum Wells
}

\author{
C. Ciano, ${ }^{1}$ M. Virgilio, ${ }^{2,}{ }^{*}$ M. Montanari,${ }^{1}$ L. Persichetti, ${ }^{1}$ L. Di Gaspare,${ }^{1}$ M. Ortolani, ${ }^{3}$ \\ L. Baldassarre, ${ }^{3}$ M.H. Zoellner, ${ }^{4}$ O. Skibitzki, ${ }^{4}$ G. Scalari, ${ }^{5}$ J. Faist, ${ }^{5}$ D.J. Paul, ${ }^{6}$ M. Scuderi, ${ }^{7}$ \\ G. Nicotra, ${ }^{7}$ T. Grange ${ }^{8}$ S. Birner, ${ }^{8}$ G. Capellini, ${ }^{1,4}$ and M. De Seta ${ }^{1}$ \\ ${ }^{1}$ Dipartimento di Scienze, Università Roma Tre, V.le G. Marconi 446, I-00146 Rome, Italy \\ ${ }^{2}$ Dipartimento di Fisica "E. Fermi, "Università di Pisa, Largo Pontecorvo 3, I-56127 Pisa, Italy \\ ${ }^{3}$ Dipartimento di Fisica, Università di Roma "La Sapienza", Piazzale A. Moro 2, I-00185 Rome, Italy \\ ${ }^{4}$ IHP - Leibniz-Institut für innovative Mikroelektronik, Im Technologiepark 25, D-15236 Frankfurt (Oder), \\ Germany \\ ${ }^{5}$ Institute for Quantum Electronics, ETH Zürich, Zürich, Switzerland \\ ${ }^{6}$ School of Engineering, University of Glasgow, Rankine Building, Oakfield Avenue, Glasgow G12 8LT, \\ United Kingdom \\ ${ }^{7}$ Istituto per la Microelettronica e Microsistemi (CNR-IMM), VIII strada 5, I-95121 Catania, Italy \\ ${ }^{8}$ nextnano GmbH, Südmährenstr. 21, D-85586 Poing, Germany
}

(Received 24 September 2018; revised manuscript received 2 December 2018; published 2 January 2019)

Theoretical predictions indicate that the $n$-type $\mathrm{Ge} / \mathrm{Si}-\mathrm{Ge}$ multi-quantum-well system is the most promising material for the realization of a $\mathrm{Si}$-compatible $\mathrm{THz}$ quantum cascade laser operating at room temperature. To advance in this direction, we study, both experimentally and theoretically, asymmetric coupled multi-quantum-well samples based on this material system, that can be considered as the basic building block of a cascade architecture. Extensive structural characterization shows the high material quality of strain-symmetrized structures grown by chemical vapor deposition, down to the ultrathin barrier limit. Moreover, THz absorption spectroscopy measurements supported by theoretical modeling unambiguously demonstrate inter-well coupling and wavefunction tunneling. The agreement between experimental data and simulations allows us to characterize the tunneling barrier parameters and, in turn, achieve highly controlled engineering of the electronic structure in forthcoming unipolar cascade systems based on $n$-type Ge/Si-Ge multi-quantum-wells.

DOI: 10.1103/PhysRevApplied.11.014003

Wavefunction engineering in semiconductor heterostructures has led to the development of quantum electronic and photonic devices based on electron tunneling phenomena, such as quantum cascade lasers (QCL) [1-3] and resonant tunneling diodes (RTD) [4,5]. At the moment, these widely employed devices are based on compound semiconductors belonging to the III-V elemental group (e.g., GaAs), and most of them exploit electron transport in the conduction band ( $n$-type doping), which has a band structure considerably simpler than the valence band. Beyond QCLs, other approaches to terahertz generation employing electron wavefunction tunneling through quantum wells include millimeter wave-pumped superlattices [6] and infraredpumped quantum fountain structures [7,8]. Recently, $n$ type $\mathrm{Ge} / \mathrm{Si}-\mathrm{Ge}$ heterostructures have been identified as a promising material system to realize CMOS-compatible light emitters leveraging on intersubband (ISB) transitions in the $\mathrm{THz}$ range and operating at (or close to) room

*michele.virgilio@unipi.it temperature [9-11]. In particular, the recent observation of ISB-transition signatures in the 4-12 THz range, featuring long nonradiative lifetimes of tens of picoseconds at a relatively high temperature (approximately $100 \mathrm{~K}$ ) [8,12-15], suggests that this material system could enable the fabrication of a Si-Ge-based THz QCL. Besides the great advantage of compatibility with the standard CMOS technology, the use of $\mathrm{Ge} / \mathrm{Si}-\mathrm{Ge}$ could also potentially overcome severe limitations of the present THz-QCL technology based on III-V compounds: (i) the weaker electron-phonon interaction in nonpolar semiconductors could increase the device operating temperature, currently limited to approximately $200 \mathrm{~K}$ [16]; (ii) the absence of a spectral range of forbidden propagation (Reststrahlen band) in groupIV semiconductors enables larger emission bandwidth for THz QCLs, well above the current limit of approximately $5 \mathrm{THz}$ [17]; (iii) the absence of a strong frequency dependence of the refractive index in group-IV materials could provide the ideal support for $\mathrm{THz}$ frequency combs without dispersion compensation [18-21]. 
In the pathway toward the realization of a Si-Ge-based QCL device, several issues must be still addressed. The growth has to be optimized in order to achieve (i) active epitaxial layers being strain-compensated, relatively thick, and of high crystalline quality; (ii) a high degree of reproducibility in the composition profile of the ultrathin barriers, as required to control the electronic spectrum, the tunneling rates, and the electron subband lifetime; (iii) sharp heterointerfaces to minimize the detrimental impact of interface roughness (IFR) scattering on the QCL gain [22]. Moreover, the development of reliable theoretical tools to model the ISB electronic dynamics in electrically biased structures beyond the naive rate equation approach is another mandatory requirement for the effective design and optimization of the active region stack [23]. This calls for detailed knowledge of the actual material parameters for the Si-Ge system, which can only be obtained through well-designed experiments, supported by an appropriate framework for the theoretical description in a multivalley nonpolar material system of the coupling between electronic states in adjacent quantum structures, tunneling rates, and state lifetimes. To this end, a pair of asymmetric coupled quantum wells (ACQWs), as the minimal building blocks of a QCL architecture, constitutes the ideal exemplar to investigate the dependence of the quantum-confined electron states on the heterostructure properties. By tuning the barrier thickness and the relative width of the two wells, one can modulate the energy of the ISB transitions (ISBT) and the spatial property of the excited electronic states, whose wavefunction can be designed with very different degrees of delocalization over the two wells. Their properties can be investigated by absorption spectroscopy, thanks to the asymmetric character of the coupled wells, which allows ISB transitions from the fundamental level to both the first- and the second-excited states, originating from the interaction of the two levels present in the isolated wells.

After having first observed ISBT in decoupled $\mathrm{Ge} / \mathrm{Si}-\mathrm{Ge}$ multi-quantum-well (MCQW) heterostructures [12-15,24,25], in this work, we perform the subsequent logical step toward a QCL design, unambiguously demonstrating electronic wavefunction tunneling between adjacent $n$-type strain-compensated $\mathrm{Ge} / \mathrm{Si}_{0.2} \mathrm{Ge}_{0.8}$ ACQWs, grown by chemical vapor deposition (CVD). Using FTIR spectroscopy coupled with numerical calculations [13] as a probe for the interwell coupling, we extract relevant information on dipole matrix elements, barrier height, and level broadening due to interface scattering [12,13,24-26]. Moreover, thanks to a thorough structural analysis, we demonstrate that the CVD growth of MQW heterostructures on Si wafers combines high reproducibility with the feasibility of the ultrathin barrier thickness required for tunneling transport, as well as a full control of the in-plane lattice parameter, a mandatory prerequisite to precisely fulfill the strain-symmetrization conditions. It follows that very thick MQW tunneling structures can be realized, avoiding strain relaxation as required to confine the optical mode in the active region of a QCL operating in the THz range.

\section{METHODS}

Five strain-compensated $\mathrm{Ge} / \mathrm{Si}_{0.2} \mathrm{Ge}_{0.8}$ multi-ACQW samples consisting of large and narrow Ge wells separated by a tunneling barrier with thicknesses $w_{L}, w_{t}$, $b_{t}$, respectively, are grown by ultrahigh vacuum CVD at $500{ }^{\circ} \mathrm{C}$ using ultrapure germane and silane without carrier gases (see Table I and top of Fig. 1). This stack, embedded between thick $\mathrm{Si}_{0.2} \mathrm{Ge}_{0.8}$ barrier layers $\left(b_{L}\right)$, which avoids coupling between the adjacent modules, is periodically repeated 20 times. To make ISB transitions in the conduction band observable by FTIR absorption spectroscopy, a $10-\mathrm{nm}$-wide region at the center of the large well is codoped by phosphine resulting in an active phosphorous donor density of approximately $7 \times 10^{17} \mathrm{~cm}^{-3}$. A dopant diffusion profile featuring a density reduction of 1 decade $/ 10 \mathrm{~nm}$ is measured by secondary ion mass spectroscopy on calibration samples (not shown). The active quantum-well stack is grown on a reverse-graded $\mathrm{Si}_{1-\mathrm{x}} \mathrm{Ge}_{\mathrm{x}}$ virtual substrate (RG VS) on $\mathrm{Ge} / \mathrm{Si}(001)$ substrates, ending with a $1.5-\mu \mathrm{m}$-thick constant composition $\mathrm{Si}_{0.13} \mathrm{Ge}_{0.87}$ layer (details on the specific RG VS can be found in Ref. [12]). The reacting gas pressure is $1.2 \mathrm{mTorr}$, resulting in a growth rate of $6.5 \mathrm{~nm} / \mathrm{min}$ for the wells and $4.5 \mathrm{~nm} / \mathrm{min}$ for the barriers. By using these process parameters, we can carefully control the deposited thickness in the subnanometer range and in a repeatable way, as evidenced by STEM performed in a (Cs)probe-corrected JEOL JEM ARM200CF. In order to get $Z$-contrast sensitiveness, STEM images are acquired with an annular dark-field (ADF) detector. A complementary high-resolution XRD analysis is performed using a Rigaku SmartLab with a rotating anode and line-focus geometry featuring a $\mathrm{Ge}(400) \times 2$ channel-cut beam collimator and a $\mathrm{Ge}(220) \times 2$ analyzer crystal.

Absorption spectra are measured at a temperature of $T=10 \mathrm{~K}$ in side-illuminated single-pass waveguide configuration with an evacuated FTIR spectrometer (Bruker Vertex 70v), equipped with a He-flow cryostat and an automated lithographic polarizer rotator. We use a surfaceplasmon waveguide in which light enters and exits from the side facets of the sample cut at $70^{\circ}$ with the growth plane, and the top facet close to the ACQWs is coated with a metal bilayer $(\mathrm{Ti} / \mathrm{Au}, 10 \mathrm{~nm} / 80 \mathrm{~nm})$. In this configuration, the electric field of the radiation propagating through the QW layers is TM polarized and then almost parallel to the ISB dipole. In order to filter out polarizationindependent features not related to ISB transitions, such as those due to the dopant absorption in the Si substrate, we evaluate the dimensionless absorption coefficient $\alpha_{2 \mathrm{D}}(\omega)$ associated with each QW using the dichroic transmission 
TABLE I. Thickness in nm of the large (narrow) well $w_{L}\left(w_{t}\right)$, and of the thin (thick) barrier $b_{t}\left(b_{L}\right)$ for S1-S5 samples. Nominal $\left(D_{\text {nom }}^{\mathrm{SL}}\right)$ and measured $\left(D_{\mathrm{XRD}}^{\mathrm{SL}}\right)$ superlattice period is also reported; $x_{\mathrm{Ge}}$ is the Ge content measured in the thin barrier and the sheet-carrier density $n_{2 \mathrm{D}}$ in units of $10^{11} \mathrm{~cm}^{-2}$, estimated from FTIR absorption spectra following the procedure reported in Ref. [13].

\begin{tabular}{lcccccccc}
\hline \hline & $w_{L}(\mathrm{~nm})$ & $b_{t}(\mathrm{~nm})$ & $w_{t}(\mathrm{~nm})$ & $b_{L}(\mathrm{~nm})$ & $D_{\mathrm{nom}}^{\mathrm{SL}}(\mathrm{nm})$ & $D_{\text {XRD }}^{\mathrm{SL}}(\mathrm{nm})$ & $\mathrm{x}_{\mathrm{Ge}}\left(10^{11} \mathrm{~cm}^{-2}\right)$ & $n_{2 \mathrm{D}}\left(10^{11} \mathrm{~cm}^{-2}\right)$ \\
\hline S1 & 12 & 2.3 & 5 & 21 & 40.3 & 41.6 & 0.87 & 7.2 \\
S2 & 13 & 2.3 & 5 & 21 & 41.3 & 42.8 & 0.87 & 7 \\
S3 & 11.3 & 2.3 & 5 & 21 & 39.6 & 40.6 & 0.87 & 7.8 \\
S4 & 12 & 3.3 & 5 & 21 & 41.3 & 42.4 & 41 & 0.81 \\
S5 & 12 & 4 & 5 & 21 & 42 & 43 & 4.6 \\
\hline \hline
\end{tabular}

spectra $T(\omega)=T_{\mathrm{TM}}(\omega) / T_{\mathrm{TE}}(\omega)$, from which we also estimate the sheet carrier density $n_{2 \mathrm{D}}$ [13].

To design the ACQW samples, calculate their energy levels and electron wave-functions, and to simulate the optical spectra, we use a multivalley self-consistent Schrödinger-Poisson solver with parabolic subband dispersion. In this model, the band alignments of the $L, \Delta$, and $\Gamma$ conduction minima of a biaxially strained $\mathrm{Si}_{1-x} \mathrm{Ge}_{x}$ layer grown on a relaxed $\mathrm{Si}_{1-y} \mathrm{Ge}_{y}$ substrate are evaluated according to Ref. [13], where the interested reader can also find the values of the material parameters used in the present calculations. The confinement mass and density of states are calculated, updating the Ge longitudinaland transverse-mass values to $m_{l}=1.59 m_{0}$ and $m_{t}=0.093$ $m_{0}$, as recently reported in Ref. [27]. The electronic states are estimated self-consistently in the Schrödinger-Poisson iterative scheme, taking into account ionized impurities and contributions to the Hartree potential from electrons at the $L, \Delta$, and $\Gamma$ valleys. Exchange-correlation effects are included in the local density approximation [28] only for the $L$ electrons, which at low temperature represent the large majority of the carriers. In the evaluation of the optical spectra, the impact of $\Delta_{2}$ states has been neglected due to their spatial separation from $L$ states and also because their presence has never been observed in FTIR data acquired in the past from a single $\mathrm{QW}$ designed with the excited $L$ subband both in or below the energy interval where $\Delta_{2}$ states are located [13]. Finally, we notice that, while more advanced computational methods such as density matrix or nonequilibrium Green's functions are certainly mandatory to model electron transport in $\mathrm{Ge} / \mathrm{Si}-\mathrm{Ge}$ cascade heterostructures, our simple multivalley effective mass approach is sufficient to interpret the ISBT features of the FTIR spectra and to validate the set of material parameters proposed here.

\section{STRUCTURAL CHARACTERIZATION}

In Fig. 1(a), we show a high-resolution ADF STEM image of a sample, grown for calibration purposes, in which we deposit alternated $\mathrm{Si}_{0.2} \mathrm{Ge}_{0.8}$ and Ge layers with different thicknesses (dark and bright stripes, respectively). A quantitative analysis of the image clearly indicates the presence of Si-Ge intermixing at the interfaces, resulting in a broadening of the well-barrier profile over a length scale of approximately $0.8 \mathrm{~nm}$. This effect is obviously more relevant in thin layers (thickness $<2.5 \mathrm{~nm}$ ), well matching the results of Bashir et al. on nominally identical Ge/Si-Ge MQWs [29], and thus leading to a thicknessdependent lowering of the confining potential. Nonetheless, taking this intermixing effect into account, we obtain a reasonable agreement of the measured layer thickness with the nominal one [see inset of Fig. 1(a)]. An upper limit of $0.4 \mathrm{~nm}$ for the root-mean-square IFR can be derived from the high-resolution analysis of several heterointerfaces.

In Fig. 1(b), we show the ADF STEM of sample S4, where one can clearly observe the abruptness of the interfaces and thickness reproducibility (within approximately $2 \%$ ) of the growth in each of the ACQW modules. The high reproducibility and quality of the heterostructures can be also seen from the XRD data reported in Fig. 1(c), where we show the reciprocal space map (RSM) around the (224) reflections acquired on sample S3. The RSM plot indicates that the entire superstructure is lattice matched to the Ge-Si buffer layer [30], which exhibits a stoichiometry of $x=0.87$ and a slightly residual tensile strain of $\epsilon_{\|}=0.20 \%$, which can be attributed to the difference of the coefficient of thermal expansion existing between the $\mathrm{Si}$ substrate and the RG VS [31]. The measured in-plane lattice parameter $a_{\|}=5.6373 \AA$ corresponds to a cubic Si-Ge layer with an equivalent composition $x_{\mathrm{eq}} \sim 0.92$, which, in turn, sets the condition for a fully coherent superlattice. From the superlattice-fringe analysis, we obtain an average Ge composition for the ACQW barriers of sample S4 $x_{\mathrm{Ge}}=0.81 \pm 0.01$ (see Table I). The superlattice period $D_{\mathrm{XRD}}^{\mathrm{SL}}$, calculated for the whole sample set, are also reported in Table I. The values measured by XRD agree with the ADF STEM results and are equal to the nominal values within $2.5 \%$. The effective Ge content determined from the superlattice period measurement is approximately 0.91, thus confirming the matching condition for strain balancing within the experimental error.

\section{WAVEFUNCTION ENGINEERING}

The electron wavefunctions in ACQWs are very sensitive to the properties of the tunneling barrier as well 

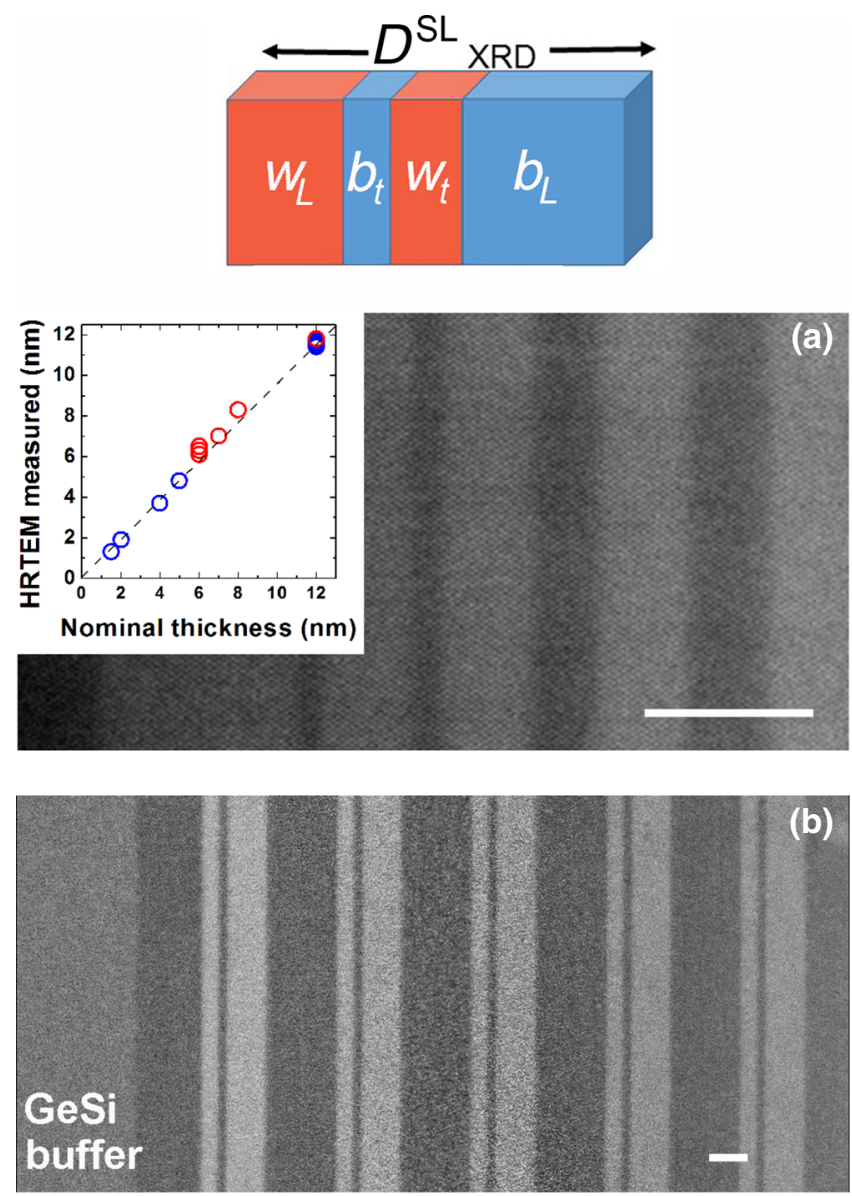

(c)

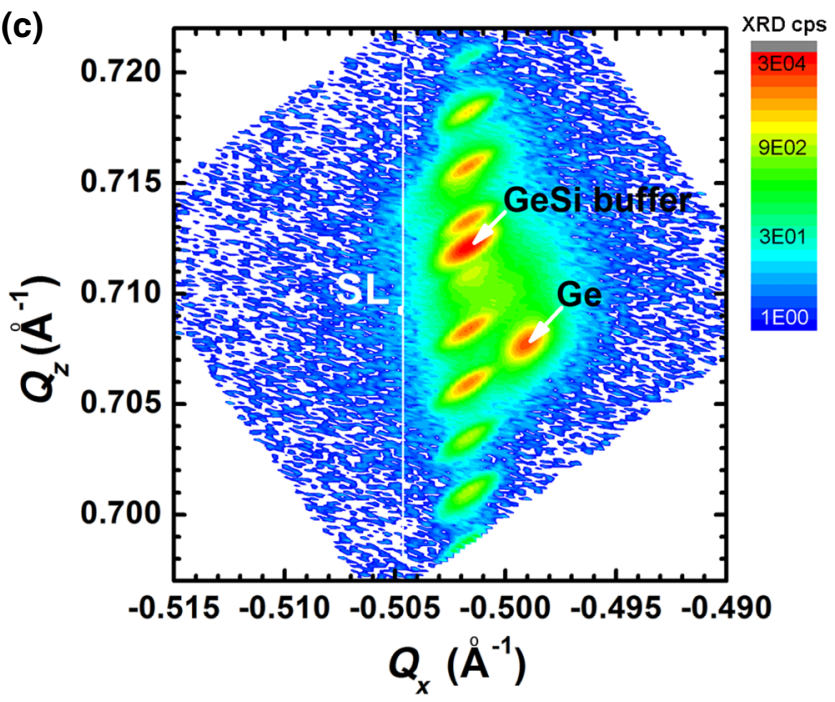

FIG. 1. (Top) A schematic diagram of an ACQW module with definition of the relevant thicknesses (see Table I). (a) $Z$-contrast STEM micrograph of a $\mathrm{Ge} / \mathrm{Si}_{0.2} \mathrm{Ge}_{0.8}$ calibration sample (alloy material appears darker); in the inset, the thickness of the Ge well (red) and Si-Ge barrier (blue) measured by STEM vs nominal thickness. The narrowest Si-Ge barrier is $1.6 \mathrm{~nm}$ thick. (b) $Z$ contrast STEM image of sample S4. (c) XRD RSM around the (224) reflections of Si-Ge for sample S3. Scale bars are $10 \mathrm{~nm}$ in panels (a) and (b). as to the energy detuning of the isolated-well levels, which hybridize to form states extending over both the interacting wells. To disentangle these two effects, in the following, we compare ISBT spectra acquired on a sample series featuring the same well thickness but different $b_{t}(\mathrm{~S} 1, \mathrm{~S} 4, \mathrm{~S} 5)$ and on a sample series with the same $b_{t}$ but different $w_{L}$ (S3, S1, S2). In order to interpret the experimental ISB absorption spectra, we employ a self-consistent multivalley effective mass model [13], with the measured structural parameters as inputs. As a starting point, we calculate in Fig. 2 the fundamental $\left(E_{0}\right)$, first-excited $\left(E_{1}\right)$, and secondexcited $\left(E_{2}\right)$ electron states of asymmetric quantum wells with $w_{t}=5 \mathrm{~nm}$ as a function of the large well width $w_{L}$, in the uncoupled $\left(b_{t}=10 \mathrm{~nm}\right)$ and in the coupled regimes $\left(b_{t}=2.3 \mathrm{~nm}\right)$. In order to take into account the unavoidable $\mathrm{Si}-\mathrm{Ge}$ intermixing of real samples, $x_{\mathrm{Ge}}$ has been set to 0.81 and 0.87 (implying different barrier heights) for the uncoupled and coupled regimes, respectively (see Table I). Figure 2(a) displays the energy of the states in the uncoupled regime as a function of $w_{L}:$ at $w_{L} \approx 14 \mathrm{~nm}$, the second level of the large well aligns to the first level of the narrow well, whose confinement energy remains almost unaffected by the presence of the large well. For smaller (larger) values of $w_{L}$, the first-excited level of the uncoupled system $E_{1}$ has a completely "narrow-well" ("large-well") character, as demonstrated by the square of the electron wavefunctions reported in Figs. 2(b) and 2(c), where electronic levels have been calculated for $w_{L}=8$ and $w_{L}=22 \mathrm{~nm}$, respectively. The fundamental state $E_{0}$ is always confined in the large well.

Figures 2(d)-2(f) instead display, respectively, the energy of the states and the square of the electron wavefunctions calculated for $w_{L}=8 \mathrm{~nm}$ and $w_{L}=22 \mathrm{~nm}$ in the coupled configuration $\left(b_{\mathrm{t}}=2.3 \mathrm{~nm}\right)$. Upon comparing Figs. 2(e) and 2(f) with their counterparts in the uncoupled case, i.e., Figs. 2(b) and 2(c), it is apparent that the wavefunctions of $E_{1}$ and $E_{2}$, although featuring the same main character, now have a non-negligible amplitude in both wells due to the interwell coupling resulting from quantum tunneling through the barrier. State hybridization between the fundamental level in the narrow well and the first-excited state in the large well peaks when their energy detuning approaches zero, resulting in the typical level-repulsion behavior evident in Fig. 2(d), from which we estimate, at the crossing point $w_{L} \approx 14 \mathrm{~nm}$, an anticrossing energy of approximately $12 \mathrm{meV}$.

We now turn to the calculation of the ISB transition energies and oscillator strengths. In the top panels of Fig. 3, the solid curves represent the calculated energies $E_{01}=E_{1}-E_{0}$ and $E_{02}=E_{2}-E_{0}$ as a function of $w_{L}$ for uncoupled [Fig. 3(a)] and coupled [Fig. 3(c)] ACQWs. The position of the related resonances expected in the absorption spectra are instead shown as dotted curves. The energy difference between the absorption resonance and 

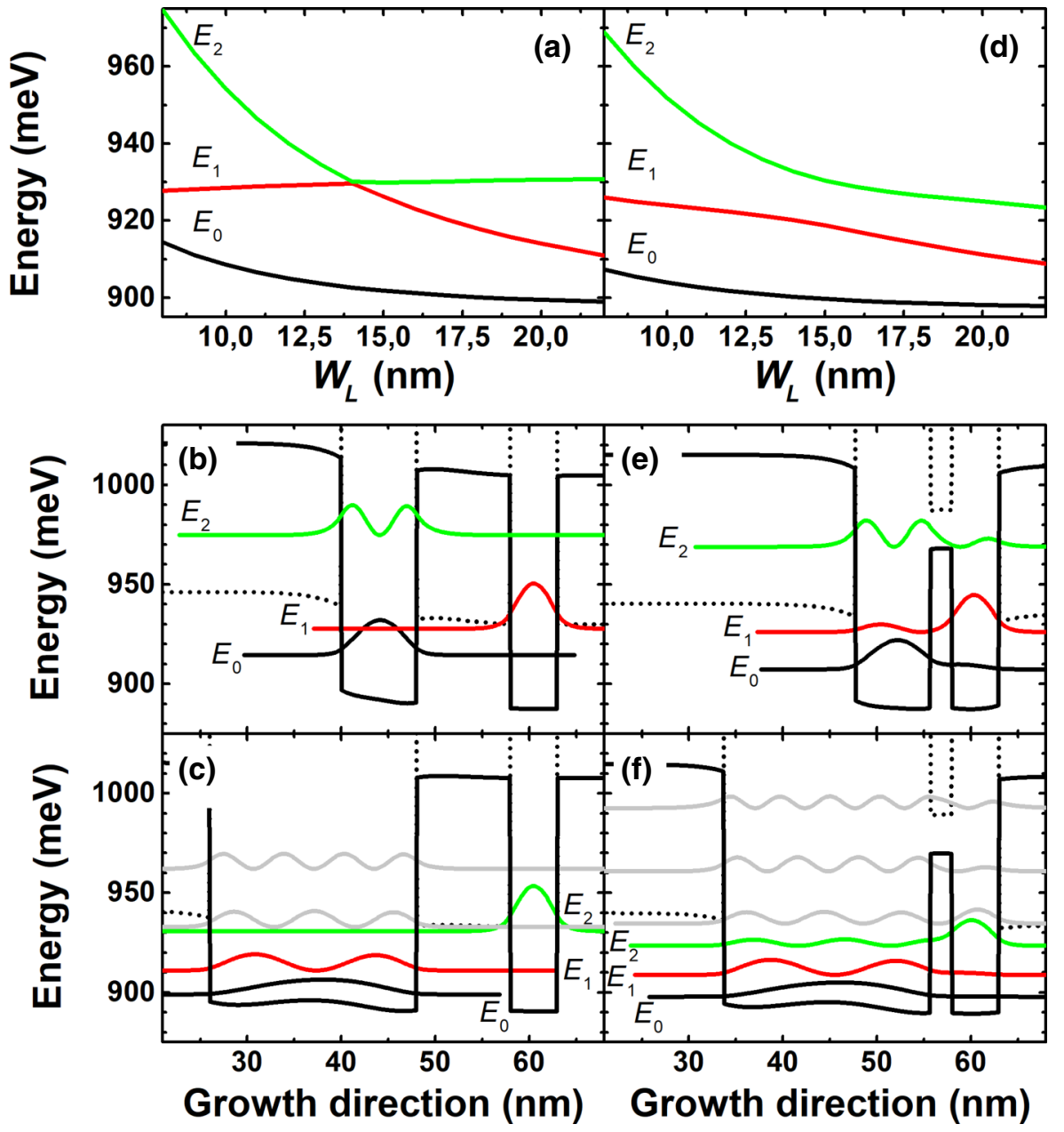

FIG. 2. Energy of the fundamental, first-excited, and second-excited electron states $\left(E_{0}, E_{1}\right.$, and $E_{2}$, respectively) calculated for (a) uncoupled, $b_{t}=10 \mathrm{~nm}$ and $x_{\mathrm{Ge}}=0.81$, and (d) coupled, $b_{t}=2.3 \mathrm{~nm}$ and $x_{\mathrm{Ge}}=0.87$, ACQWs as a function of $w_{L}$. Electron energy and squared wavefunction at $w_{L}=8$ and $w_{L}=22 \mathrm{~nm}$ for uncoupled (b),(c) and coupled (e),(f) ACQWs (solid and dotted black curves represent the $L$ and $\Delta_{2}$ band profiles, respectively). The potential drop across the ACQW period in plot (b) is related to static charge effects associated with the presence of a dopant tail in the tunneling barrier and in the thin well.
$E_{01}, E_{02}$ stems from the depolarization shift effect induced by the rather high free-electron density resulting from the local dopant concentration $\left(n=7 \times 10^{17} \mathrm{~cm}^{-3}\right)$ extending over a width of about $10 \mathrm{~nm}[13,25,32]$. The oscillator strengths of the ISB transitions $f_{01}$ and $f_{02}$ are calculated for the uncoupled case [Fig. 3(b)] and for the coupled case [Fig. 3(d)].

In the uncoupled regime [Figs. 3(a) and 3(b)], only the transition involving the second level of the large well has a nonzero oscillator strength, because the fundamental state is always localized in the large well and has no overlap with the first level of the narrow well. This transition corresponds to $E_{02}$ for $w_{L}<14 \mathrm{~nm}$ and to $E_{01}$ for $w_{L}>14 \mathrm{~nm}$. As a consequence, the effect of the depolarization shift, which can be seen only in transitions between states with non-negligible spatial overlap, is absent in $E_{01}$ for $w_{L}<14 \mathrm{~nm}$ and in $E_{02}$ for $w_{L}>14 \mathrm{~nm}$. In the coupled ACQW [Figs. 3(c) and 3(d)], both transitions are active due to level hybridization and, for $w_{L}<14 \mathrm{~nm}$, they acquire comparable oscillator strengths. A peak value of $f_{02} / f_{01} \sim 0.9$ is predicted at $w_{L} \approx 10 \mathrm{~nm}$.

\section{INTERSUBBAND ABSORPTION MEASUREMENTS}

The conceptual framework introduced in the previous section is now used to interpret the experimental ISB absorption spectra of $\mathrm{Ge} / \mathrm{Si}-\mathrm{Ge}$ ACQW samples measured by FTIR spectroscopy at $T=10 \mathrm{~K}$. At this temperature, excited states are empty, so that only transitions having $E_{0}$ as an initial level are active. The experimentally determined two-dimensional (2D) absorption coefficient spectra $\alpha_{2 \mathrm{D}}(\omega)$, reported in Figs. 4(a) and 5(a), are fitted with the sum of two Lorentzian peaks (identified with indices $i=1$, 2 ), each having, as free parameters, the integrated spectral weight $A_{i}$, the half-width-at-half-maximum $\Gamma_{I}$, and the peak energy $E_{0 i}^{\text {abs }}$. Experimental data for $\alpha_{2 \mathrm{D}}(\omega)$ are compared with ISBT theoretical absorption spectra obtained with a transition-dependent Lorentzian width, set equal to the measured ones [see Figs. 4(b) and 5(b)]. To this aim, we note that the $1 / \omega$ functional dependence, present in the expression used to model the ISBT absorption coefficient, is filtered out in the experimental spectra since, in this case, $\alpha_{2 \mathrm{D}}(\omega)$ has been estimated from the dichroic transmittance. The small overestimation of the experimental data in the 


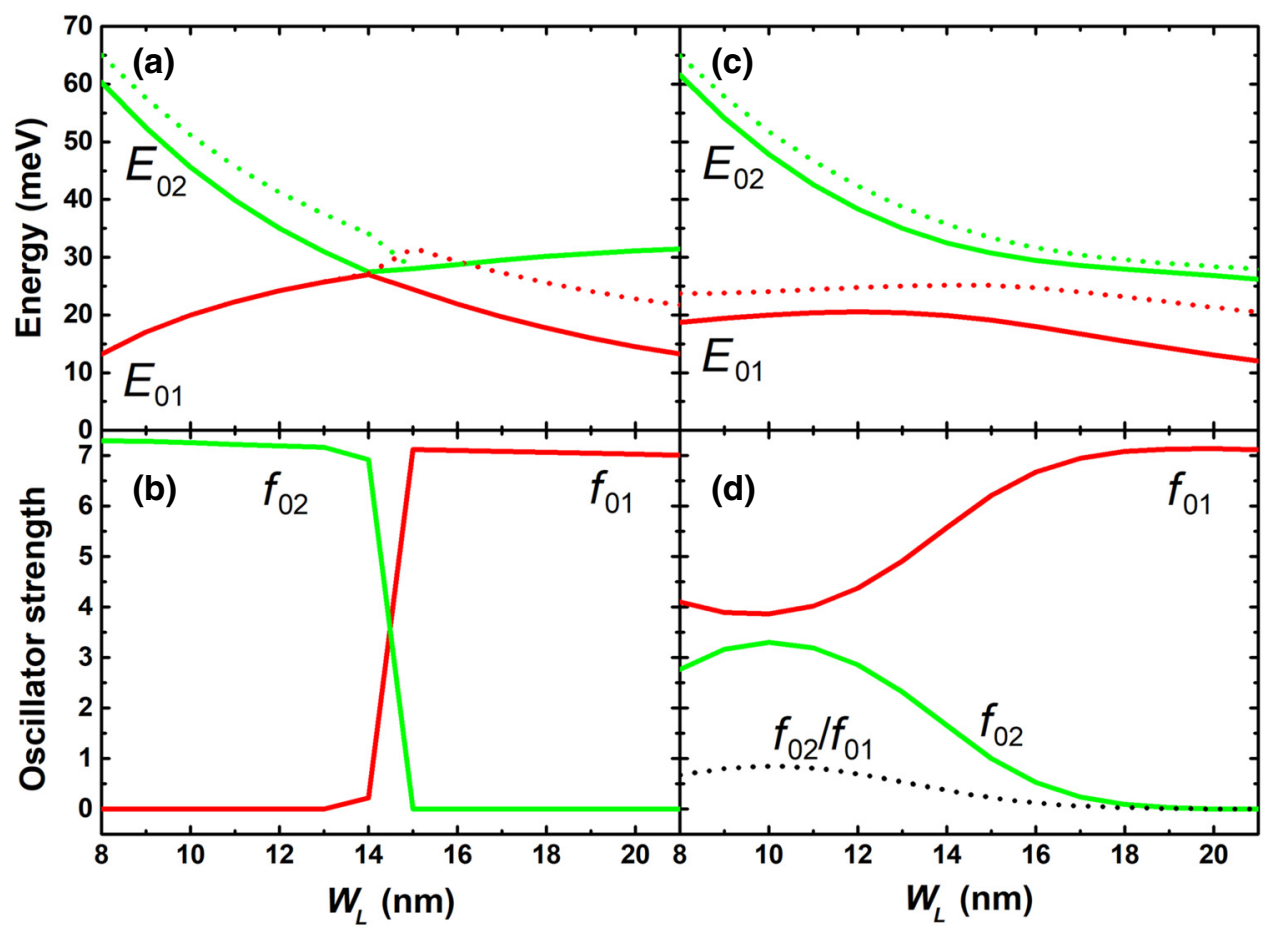

FIG. 3. Transition energy and oscillator strength as a function of $w_{L}$, calculated (a),(b) for $b_{t}=10 \mathrm{~nm}$ and $x_{\mathrm{Ge}}=0.81$ and (c),(d) for $b_{t}=2.3 \mathrm{~nm}$ and $x_{\mathrm{Ge}}=0.87$. Solid red (green) curve refers to the $E_{01}\left(E_{02}\right)$ transition. In panels (a),(c), the energy of the calculated $E_{01}$ and $E_{02}$ absorption resonances are represented by dotted curves. In panel (d), the ratio between the two oscillator strengths is also reported. The oscillator strength of each transition varies between 0 and $1 / m^{*}$ where $m^{*}=0.135$ in electron-mass units is the electron effective mass along the confinement direction, resulting from the values for the $L$ inverse mass tensor reported in Ref. [27]. low-energy region of the ISBT spectra can be attributed to this effect.

In Fig. 4, we analyze ACQWs having the same $w_{L}=12 \mathrm{~nm}$ and $b_{t}$ ranging between $2.3 \mathrm{~nm}$ and $4 \mathrm{~nm}$ (samples S1, S4, S5). The experimental spectra are very well reproduced by theory. The energy separation $\Delta E^{\mathrm{abs}}=$ $E_{02}^{\text {abs }}-E_{01}^{\text {abs }}$ plotted in Fig. 4(c) decreases for increasing $b_{t}$ due to the closing of the anticrossing energy gap. Correspondingly, since for $w_{L}=12 \mathrm{~nm}$ the $E_{2}$ level of the ACQW system mainly has the character of the first-excited state in the large well, we observe an increase of the spectral weight ratio $A_{2} / A_{1}$ [Fig. 4(d)], in agreement with the fact that in the thick-barrier limit, the $E_{01}$ transition is completely suppressed for small values of $w_{L}$ [see Fig. 3(b)]. Note, however, that $b_{t}=4 \mathrm{~nm}$ differs significantly from the uncoupled regime since the spectral weights of the two peaks are still comparable. Indeed, the $E_{2}$ and $E_{1}$ wavefunctions [see Fig. 4(e)] indicate that the excited states at $b_{t}=4 \mathrm{~nm}$ are still delocalized in both wells, due to the fact that, for $w_{L}=12 \mathrm{~nm}$, their energy separation in

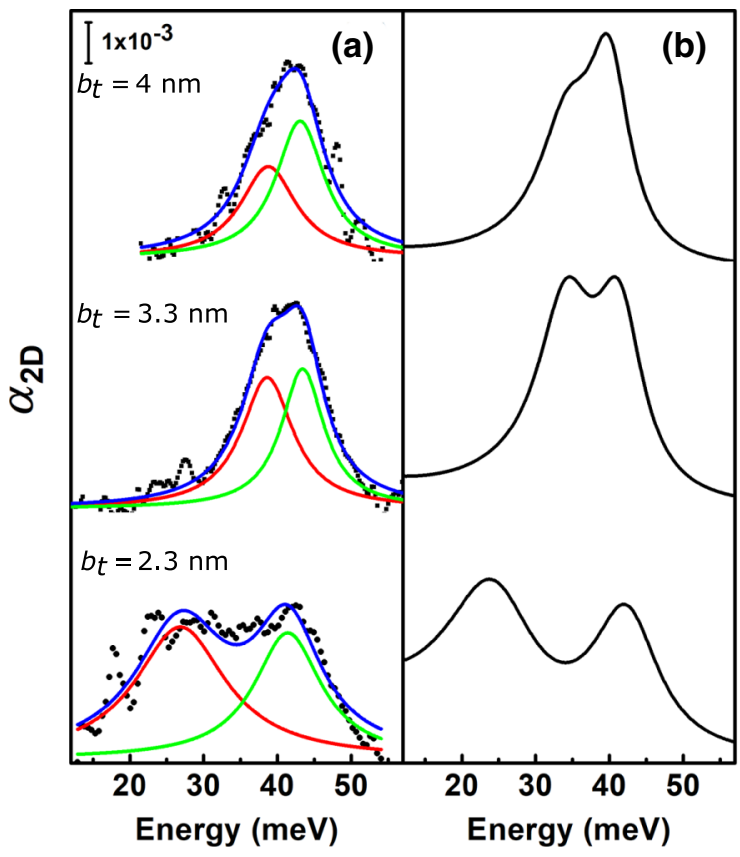

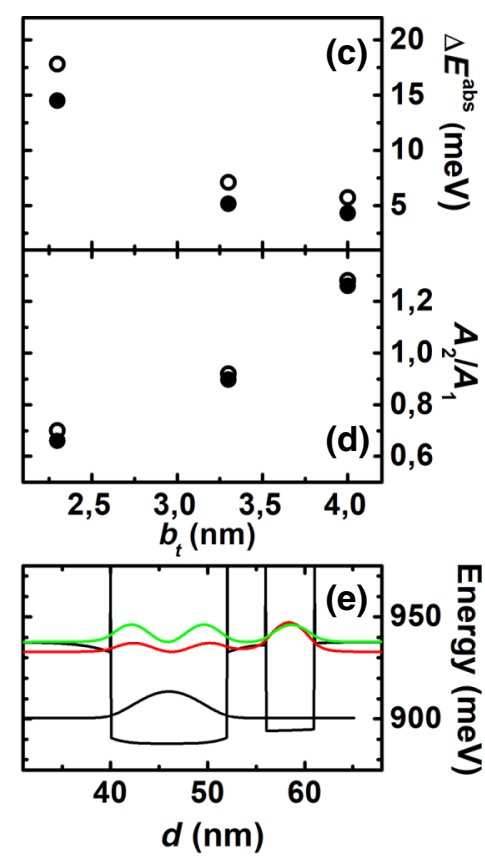

FIG. 4. (a) Dots: experimental ISB absorption spectra measured by FTIR of samples S5 (top), S4 (middle), and S1 (bottom), with Lorentzian fits of the peaks centered at $E_{01}^{\text {abs }}$ and $E_{02}^{\text {abs }}$ (red and green curves, respectively). (b) Corresponding calculated spectra for $w_{L}=12 \mathrm{~nm}$ and variable thinbarrier width $b_{t}$. (c) Energy difference $E_{02}^{\text {abs }}-E_{01}^{\text {abs }}$ and (d) ratio of the integrated spectral weight of the two ISB absorption peaks, plotted vs $b_{t}$. Closed (open) symbols are used for experimental (numerical) data. (e) Calculated squared wavefunctions for sample S5. 


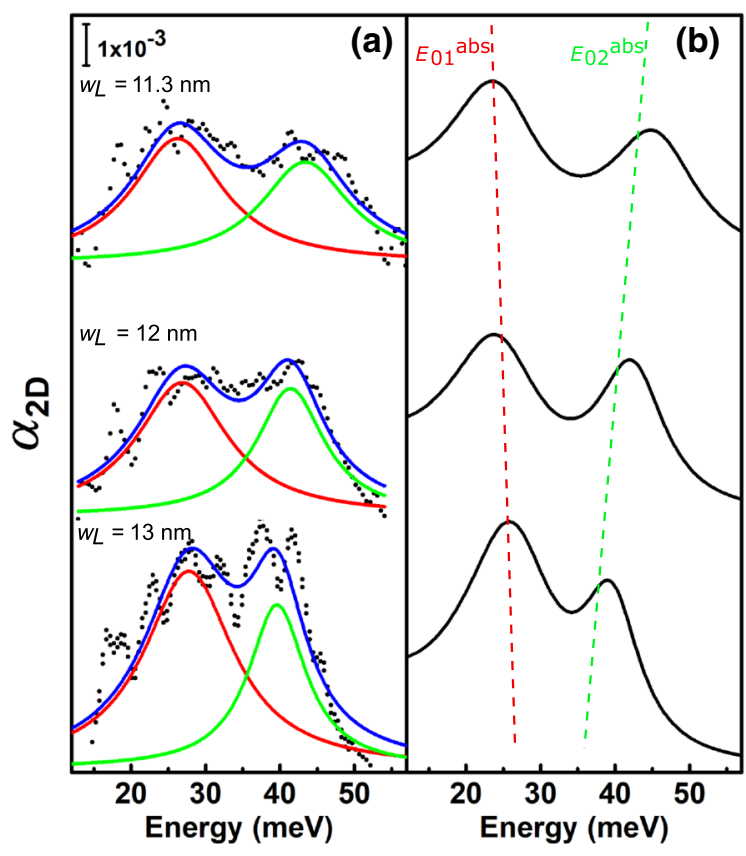

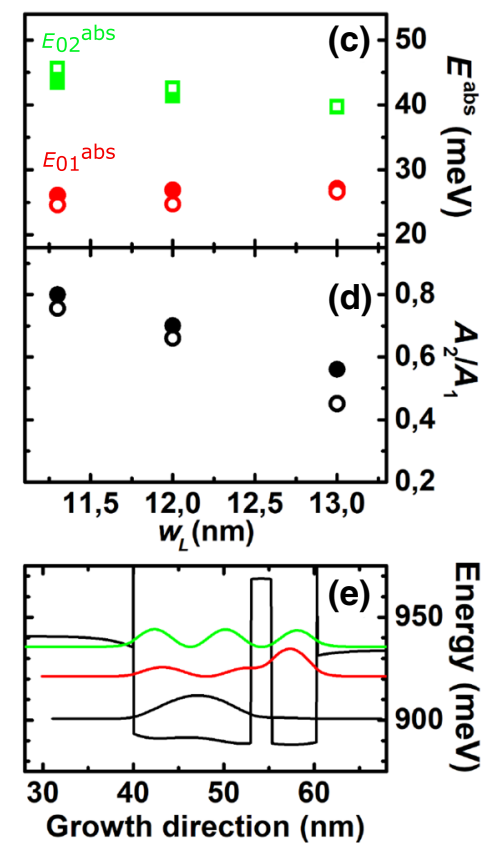

FIG. 5. (a) Dots: experimental ISB absorption spectra measured by FTIR of samples S3 (top), S1 (middle), and S2 (bottom), with Lorentzian fits of the peaks centered at $E_{01}^{\mathrm{abs}}$ and $\mathrm{E}_{02}^{\mathrm{abs}}$ (red and green curves, respectively). (b) Corresponding calculated spectra at fixed $b_{t}=2.3 \mathrm{~nm}$ for different $w_{L}$. (c) $E_{01}^{\text {abs }}$ (red symbols) and $E_{02}^{\text {abs }}$ (green symbols) and (d) ratio of the integrated spectral weight of the two ISB absorption peaks vs $w_{L}$. Closed (open) symbols are used for experimental (numerical) data. (e) Calculated squared wavefunctions for sample S2. the uncoupled limit is quite small. The absorption-peak linewidths at half maximum $\Gamma_{i}$ decrease by increasing the barrier thickness from $\Gamma_{1}=7.5 \mathrm{meV}$ and $\Gamma_{2}=5.5 \mathrm{meV}$ at $b_{t}=2.3 \mathrm{~nm}$ to $\Gamma_{1}=4.7 \mathrm{meV}$ and $\Gamma_{2}=4.1 \mathrm{meV}$ at $b_{t}=4 \mathrm{~nm}$. We attribute this trend to the reduced impact of IFR scattering on the line broadening triggered by the decreased value of the electron squared wavefunction at the interfaces $\left(E_{2}\right.$ and $E_{1}$ interact with only two interfaces when $\left.b_{t} \rightarrow \infty\right)$.

In Fig. 5, we study the interwell coupling as a function of the energy separation between the isolated well levels by comparing the experimental and theoretical absorption spectra of samples with varying $w_{L}$ and fixed $b_{t}=2.3 \mathrm{~nm}$ (samples S3, S1, S2). In reasonable agreement with the theory, we observe in Figure 5(c) that $E_{01}$ slightly increases with $w_{L}$, while $E_{02}$, involving for $w_{L}<14 \mathrm{~nm}$ a final state with a character reflecting the one of the first-excited level in the isolated large well [see Fig. 3(c)], displays a more robust decreasing trend with $w_{L}$. The trends reported in Fig. 5(c) result in the decrease of the energy separation of the two absorption peaks, $\Delta E^{\text {abs }}$, with increasing $w_{L}$, as observed in Figs. 5(a) and 5(b). Correspondingly, when $w_{L}$ increases from 11.3 to $13 \mathrm{~nm}$, the integrated spectral-weight ratio $A_{2} / A_{1}$ decreases from 0.8 to 0.6 [see Figs. 5(d) and 3(d)], since the level hybridization gets larger and then the $E_{2}\left(E_{1}\right)$ wavefunction becomes more delocalized, leaking also in the narrow (large) well, thus reducing (increasing) the $f_{02}\left(f_{01}\right)$ oscillator strength. Interestingly, the linewidth $\Gamma_{2}$ decreases for increasing $w_{L}$, and for $w_{L}=13 \mathrm{~nm}$, the peak at $E_{02}^{\text {abs }}$ becomes narrower $\left(\Gamma_{2}=4.8 \mathrm{meV}\right)$ than the peak at $E_{01}^{\text {abs }}\left(\Gamma_{1}=7.0 \mathrm{meV}\right)$. This fact can be related to the reduced amplitude of the $E_{2}$ wavefunction at the tunneling barrier interfaces with respect to the $E_{1}$ one, as can be seen in Fig. 5(e). This reduction can be explained considering that the $E_{2}\left(E_{1}\right)$ level represents the antibonding (bonding) state, resulting from the coupling of the first-excited large-well level with the fundamental narrow-well level. It follows that the reduced amplitude of the $E_{2}$ wavefunction at the tunneling barrier interfaces is due to the increase of the bondingantibonding interaction when $w_{L}$ ranges from 11.3 to $13 \mathrm{~nm}$.

As a final remark, the great accuracy reached by our modeling in terms of (i) prediction of the absorption energies, (ii) intensity of the level repulsion, and (iii) ratio of the oscillator strengths, indicates that our band alignment procedure is quite realistic, and therefore, that our work may be useful in order to definitively establish the $L$-point conduction-band offsets in the Si-Ge-heterostructure material system. For our samples, we find a discontinuity of $117 \mathrm{meV}$ for $x_{\mathrm{Ge}}=0.81$ and of $80 \mathrm{meV}$ for $x_{\mathrm{Ge}}=0.87$. We emphasize that the growth and characterization of ACQWs is crucial for this purpose, since the ISB absorption spectra of ACQWs are much more sensitive to the precise value of the barrier height than those measured in isolated wells [33]. The confirmed band offset around $100 \mathrm{meV}$ for $n$-type $\mathrm{Ge} / \mathrm{Si}-\mathrm{Ge}$ heterostructures makes them a viable alternative to nonlinear frequency generation of mid-infrared QCLs $[34,35]$ on route to a silicon-based $\mathrm{THz}$ laser.

\section{CONCLUSION}

We have grown strain-compensated $n$-type $\mathrm{Ge} / \mathrm{Si}-\mathrm{Ge}$ multi-ACQW samples, and carefully studied, experimentally and numerically, their ISB absorption spectra, providing a clear interpretative framework of the main 
experimental features. Our results demonstrate that highquality tunneling $\mathrm{Ge} / \mathrm{Si}-\mathrm{Ge}$ heterostructures for electrons at $L$ points of the Ge conduction band can be realized with great reproducibility by CVD. The modeling accuracy in reproducing experimental ISB absorption spectra guarantees high precision of design and high reliability of material parameters for future Si-Ge-based $\mathrm{THz}$ electronic and photonic devices. In particular, the study of wavefunction tunneling in ACQW samples provides reliable values for the estimation of conduction band offset, which ultimately defines the tunneling barrier height. In summary, our results represent a step forward for the realization of $\mathrm{Ge} / \mathrm{Si}-\mathrm{Ge} \mathrm{THz}$ lasers with broad far-infrared emission range, low refractive index dispersion for frequency-comb operation, and high operating temperature.

\section{ACKNOWLEDGMENTS}

This work is supported by the European Union research and innovation program Horizon 2020 under Grant No. 766719 - FLASH Project.

[1] R. Köhler, A. Tredicucci, F. Beltram, H. E. Beere, E. H. Linfield, A. G. Davies, D. A. Ritchie, R. C. Iotti, and F. Rossi, Terahertz semiconductor-heterostructure laser, Nature 417, 156 (2002).

[2] B. S. Williams, Terahertz quantum-cascade lasers, Nat. Photonics 1, 517 (2007).

[3] M. S. Vitiello, G. Scalari, B. Williams, and P. De Natale, Quantum Cascade Lasers: 20 years of challenges, Opt. Express 23, 5167 (2015).

[4] R. Tsu, L. L. Chang, G. A. Sai-Halasz, and L. Esaki, Effects of Quantum States on the Photocurrent in a Superlattice, Phys. Rev. Lett. 34, 1509 (1975).

[5] J. P. Sun, G. I. Haddad, P. Mazumder, and J. N. Schulman, Resonant tunneling diodes: Models and properties, Proc. IEEE 86, 641 (1998).

[6] M. F. Pereira, J. P. Zubelli, D. Winge, A. Wacker, A. S. Rodrigues, V. Anfertev, and V. Vaks, Theory and measurements of harmonic generation in semiconductor superlattices with applications in the $100 \mathrm{GHz}$ to $1 \mathrm{THz}$ range, Phys. Rev. B 96, 045306 (2017).

[7] F. H. Julien, J. M. Lourtioz, N. Herschkorn, D. Delacourt, J. P. Pocholle, M. Papuchon, R. Planel, and G. LeRoux, Optical saturation of intersubband absorption in $G a A s-A l_{x} G a_{1-x} A s$ quantum wells, Appl. Phys. Lett. 53, 116 (1988).

[8] D. Sabbagh, J. Schmidt, S. Winnerl, M. Helm, L. Di Gaspare, M. De Seta, M. Virgilio, and M. Ortolani, Electron dynamics in silicon-germanium terahertz quantum fountain structures, ACS Photonics 3, 403 (2016).

[9] K. Driscoll and R. Paiella, Design of n-type silicon-based quantum cascade lasers for terahertz light emission, J. Appl. Phys. 102, 093103 (2007).

[10] D. J. Paul, The progress towards terahertz quantum cascade lasers on silicon substrates, Laser Photon. Rev. 4, 610 (2010).
[11] A. Valavanis, T. V. Dinh, L. J. M. Lever, Z. Ikonic, and R. W. Kelsall, Material configurations for n-type silicon-based terahertz quantum cascade lasers, Phys. Rev. B 83, 195321 (2011).

[12] M. De Seta, G. Capellini, Y. Busby, F. Evangelisti, M. Ortolani, M. Virgilio, G. Grosso, G. Pizzi, A. Nucara, and S. Lupi, Conduction band intersubband transitions in Ge/SiGe quantum wells, Appl. Phys. Lett. 95, 051918 (2009).

[13] Y. Busby, M. De Seta, G. Capellini, F. Evangelisti, M. Ortolani, M. Virgilio, G. Grosso, G. Pizzi, P. Calvani, S. Lupi, M. Nardone, G. Nicotra, and C. Spinella, Nearand far-infrared absorption and electronic structure of Ge-SiGe multiple quantum wells, Phys. Rev. B 82, 205317 (2010).

[14] M. Ortolani, D. Stehr, M. Wagner, M. Helm, G. Pizzi, M. Virgilio, G. Grosso, G. Capellini, and M. De Seta, Long intersubband relaxation times in n-type germanium quantum wells, Appl. Phys. Lett. 99, 201101 (2011).

[15] M. Virgilio, M. Ortolani, M. Teich, S. Winnerl, M. Helm, D. Sabbagh, G. Capellini, and M. De Seta, Combined effect of electron and lattice temperatures on the long intersubband relaxation times of $G e / S i_{x} G e_{1-x}$ quantum wells, Phys. Rev. B 89, 045311 (2014).

[16] S. Fathololoumi, E. Dupont, C. W. I. Chan, Z. R. Wasilewski, S. R. Laframboise, D. Ban, A. Mátyás, C. Jirauschek, Q. Hu, and H. C. Liu, Terahertz quantum cascade lasers operating up to $\sim 200 \mathrm{~K}$ with optimized oscillator strength and improved injection tunneling, Opt. Express 20, 3866 (2012).

[17] K. Ohtani, D. Turcinkova, C. Bonzon, I.-C. BeneaChelmus, M. Beck, J. Faist, M. Justen, U. U. Graf, M. Mertens, and J. Stutzki, High performance 4.7 THz GaAs quantum cascade lasers based on four quantum wells, New J. Phys 18, 123004 (2016).

[18] D. Burghoff, T.-Y. Kao, N. Han, C. W. I. Chan, X. Cai, Y. Yang, D. J. Hayton, J.-R. Gao, J. L. Reno, and Q. Hu, Terahertz laser frequency combs, Nat. Photonics 8, 462 (2014).

[19] J. R. Freeman, L. Ponnampalam, H. Shams, R. A. Mohandas, C. C. Renaud, P. Dean, L. Li, A. Giles Davies, A. J. Seeds, and E. H. Linfield, Injection locking of a terahertz quantum cascade laser to a telecommunications wavelength frequency comb, Optica 4, 1059 (2017).

[20] A. Forrer, M. Rösch, M. Singleton, M. Beck, J. Faist, and G. Scalari, Coexisting frequency combs spaced by an octave in a monolithic quantum cascade laser, Opt. Express 26, 23167 (2018)

[21] J. Hillbrand, P. Jouy, M. Beck, and J. Faist, Tunable dispersion compensation of quantum cascade laser frequency combs, Opt. Express 43, 1746 (2018).

[22] J. B. Khurghin, Inhomogeneous origin of the interface roughness broadening of intersubband trransitions, Appl. Phys. Lett. 93, 091104 (2008).

[23] D. O. Winge, M. Franckié, and A. Wacker, Simulating terahertz quantum cascade lasers: Trends from samples from different labs, J. Appl. Phys. 120, 114302 (2016).

[24] M. Virgilio, D. Sabbagh, M. Ortolani, L. Di Gaspare, G. Capellini, and M. De Seta, Physical mechanisms of intersubband-absorption linewidth broadening in s-Ge/SiGe quantum wells, Phys. Rev. B 90, 155420 (2014). 
[25] M. De Seta, G. Capellini, M. Ortolani, M. Virgilio, G. Grosso, G. Nicotra, and P. Zaumseil, Narrow intersubband transitions in n-type $\mathrm{Ge} / \mathrm{SiGe}$ multi-quantum wells: Control of the terahertz absorption energy trough the temperature dependent depolarization shift, Nanotechnology 23, 465708 (2012).

[26] T. Unuma, M. Yoshita, T. Noda, H. Sakaki, and H. Akiyama, Intersubband absorption linewidth in GaAs quantum wells due to scattering by interface roughness, phonons, alloy disorder, and impurities, J. Appl. Phys. 93, 1586 (2003).

[27] S. Gupta, D. Nam, J. Vuckovic, and K. Saraswat, Room temperature lasing unraveled by a strong resonance between gain and parasitic absorption in uniaxially strained germanium, Phys. Rev. B 97, 155127 (2018).

[28] O. Gunnarson and B. I. Lundqvist, Exchange and correlation in atoms, molecules, and solids by the spin-densityfunctional formalism, Phys. Rev. B 13, 4274 (1976).

[29] A. Bashir, K. Gallacher, R. W. Millar, D. J. Paul, A. Ballabio, J. Frigerio, G. Isella, D. Kriegner, M. Ortolani, J. Barthel, and I. MacLaren, Interfacial sharpness and intermixing in a Ge-SiGe multiple quantum well structure, J. Appl. Phys. 123, 035703 (2018).
[30] V. S. Kopp, V. M. Kaganer, G. Capellini, M. De Seta, and P. Zaumseil, X-ray diffraction study of plastic relaxation in Ge-rich SiGe virtual substrates, Phys. Rev. B 85, 245311 (2012).

[31] G. Capellini, M. De Seta, P. Zaumseil, G. Kozlowski, and T. Schroeder, High temperature x-ray diffraction measurements on $\mathrm{Ge} / \mathrm{Si}(001)$ heterostructures: A study on the residual tensile strain, J. Appl. Phys. 111, 073518 (2012).

[32] M. Helm, Intersubband transitions in quantum wells, in Semiconductors and Semimetals, edited by H. C. Liu and F. Capasso (Academic Press, New York, 2000), pp. 1-99.

[33] P. Rauter, T. Fromherz, N. Q. Vinh, B. N. Murdin, G. Mussler, D. Grützmacher, and G. Bauer, Continuous Voltage Tunability of Intersubband Relaxation Times in Coupled SiGe Quantum Well Structures Using Ultrafast Spectroscopy, Phys. Rev. Lett. 102, 147401 (2009).

[34] Q. Y. Lu, N. Bandyopadhyay, S. Slivken, Y. Bai, and M. Razeghi, High performance terahertz quantum cascade laser sources based on intracavity difference frequency generation, Opt. Express 21, 968 (2013).

[35] S. Jung, J. H. Kim, Y. Jiang, K. Vijayraghavan, and M. A. Belkin, Terahertz difference frequency quantum cascade laser sources on silicon, Optica 4, 38 (2017). 